\title{
MERCI: Standards based exchange of component information to support e-business applications
}

\author{
Wolfgang Wilkes \\ University of Hagen, Germany \\ wolfgang.wilkes@fernuni-hagen.de
}

\begin{abstract}
Product developers need high quality information about the components which they use in their product development. The exchange of this component information is today often still in an "archaic" stage: component data hardly exists in computer sensible form, often it is distributed only in form of textual data sheets in pdf format. This paper presents the MERCI system which exploits the PLIB standard for the exchange of component data between the processing systems of manufacturers and users.
\end{abstract}

Key words: supply chain management, component information exchange, PLIB

\section{INTRODUCTION}

Today, the design and manufacturing of products cannot be done by a single company. Almost all products integrate components which are produced by other companies. The relationship between product manufacturers and their suppliers, the component manufacturers, varies from a close relationship where component manufacturers are integrated in the product development process (they produce specific components for specific products) to a loosely relationship where independent component manufacturers offer their components on the market to potential customers. The latter case can be found quite often in the area of electronic components (e.g. memories), whereas the former one is found often in the automotive industries.

In both cases, the product manufacturer is dependent on information about the components: This information is needed in the product 
development and design process. In case of close relationships, often the customer (the product developer) defines the way in which the supplier (the component manufacturer) has to deliver the data for the specific project. If, on the other hand, components are offered for the market, the component manufacturer decides himself in which format to describe his components and how to characterise them. Unfortunately, even today it is quite common that the important characteristics of electronic components are described mainly in textual form in the so called data sheets. As a result, component users (product manufacturers) have to capture this information again to use it in their design tools and corporate databases.

In this paper, the situation in the domain of electronic components is described. Here components are normally offered on the free market, and all the mentioned problems are the daily burden of engineers. But recently also the manufacturers have begun to notice that a better data organisation is essential for streamlining their own documentation and publishing processes.

The remainder of this paper is organised as follows: Section 2 gives a short description of the overall information flows for electronic component distribution. A key aspect to overcome the current problems are standards for the representation of the component information and dictionaries for the description of the semantics of data. This is the topic of section 3. Finally, section 4 presents how the IST project MERCI addresses these areas. It is illustrated how standards and dictionaries are used as the foundation of the MERCI system architecture with the goal to exchange component information between the processing systems of component manufacturers and their customers.

\section{EXCHANGE OF COMPONENT INFORMATION}

\subsection{Electronic component information supply chain}

In the supply chain of electronic components, three different actors play a role:

- Manufacturers of semiconductors normally sell their components (e.g. memory chips, processors, micro controllers) only to the biggest customers, their key accounts.

- Distributors supply components to the other customers (which still may be fairly big). Brokers provide further services, e.g. the timely provision of all the different components which are used for the production of a specific product. They also check for the availability of alternative components from other manufacturers and the prices of distributors. 
- Component users buy their components from manufacturers or distributors. The design engineers use only qualified parts which have been approved by their component experts. Qualifying may also include the selection of specific manufacturers and/or distributors, e.g. on the basis of prices, availability, quality guarantees and internal rating of the manufacturer/distributor.

\subsection{Information exchange along the supply chain}

The component description is produced by manufacturers and normally provided in form of data sheets in pdf format, i.e. as text. Unfortunately, very often this is the only usable information which the manufacturer can provide to his customers. Many manufacturers do not have a well organised database containing the relevant data about their components with the possibility to generate the paper and Web documentation. Often it is the other way round: Manufacturers notice that there is a lack of data management and start to extract data from their own data sheets.

Distributors only refer to the Web page of the manufacturer, where the datasheet can be downloaded but they do not provide further component information. Often they provide some search databases to select the parts to be purchased. These kind of databases are also provided from independent parties with connection to several distributors.

At the end users side, component information has to be transferred into the design libraries which are used by the design engineers. This is normally a manually process which includes (dependent on the company and the domain) more or less tests and evaluation steps. Thus, very often data is recaptured again by qualified engineers. To have full control about the use of components, it is important to also have access to the commercial information about the component (normally stored in ERP systems), to the use of components in product assemblies (bill of materials), etc. That means, the component information used in the various systems throughout the company has to be linked.

An improvement of this situation has to start with the data organisation of the component manufacturers. This requires the integration of all publishing processes of the manufacturer on the basis of a single database containing all information about components. The source of component data has to become a database from which documents can be generated.

A second requirement is the use of standards: Only if the data exchange can be based on well accepted standards, data provided by several manufacturers can be understood and used by the component users. This will increase the possibilities of end users to easily select between the components from different manufacturers. This comparability also requires a 
clear description of the data semantics: Only if the customer can easily understand the meaning of a parameter, he can correctly select the right component without investing big research into the selection process.

Finally, for the component users it is of importance to have tools which allow the integration of component data into their design libraries and other databases and which keep the connection between the technical data and the business data (prices, availability, etc.).

\section{STANDARDS AND DICTIONARIES}

\subsection{Overview about PLIB}

For the description of electronic components a lot of information is required. Dependent on the process which is fed, different pieces of information are needed:

- For the selection of the component, parametric data describing the external properties of the component are required.

- The integration into a CAD system is supported by the provision of a logical symbol which describes the logical connection points of the chip.

- For the physical design, the description of the physical pins and the mapping between the logical and physical pins are needed.

- For the manufacturing process, the physical description of the pins is required, for simulation of the product, specific simulation models are needed, etc.

Thus, a suite of different pieces of information is required, and a number of different standards have been developed for the various kinds of information.

As a basis for the representation of component information, MERCI uses the PLIB standard (ISO 13584). PLIB provides a dictionary concept for the description of the data semantics and it allows to represent the parametric data associated with components in various ways:

- In the explicit representation, each component is described by all its properties (similar to a table where each component is represented in a single row).

- In the implicit representation, all the components of a family are described by a set of mechanisms: Some tables describe the independent properties of the components, other tables represent dependent properties, some dependent properties are described by functions, etc. By using these mechanisms, it is possible, for instance, to factor out the common property values of a family and to specify them only once, or to specify 
that the values of some properties are computed from the values of other properties. As such, it allows to model the organisation of data sheets which very often use this kind of implicit description of data.

An example illustrating these two ways of component modelling is given in figure 2: In (a) a family of screws is described. This family is defined by a table which correlates the possible diameter and length properties, a function which computes the width of the screw from its diameter, and a rule which indicates the relationship between the load and the diameter. On the other hand, for more complex components which do not exist in such a big number of variants, it is much easier to represent each component in a single row as illustrated in (b).

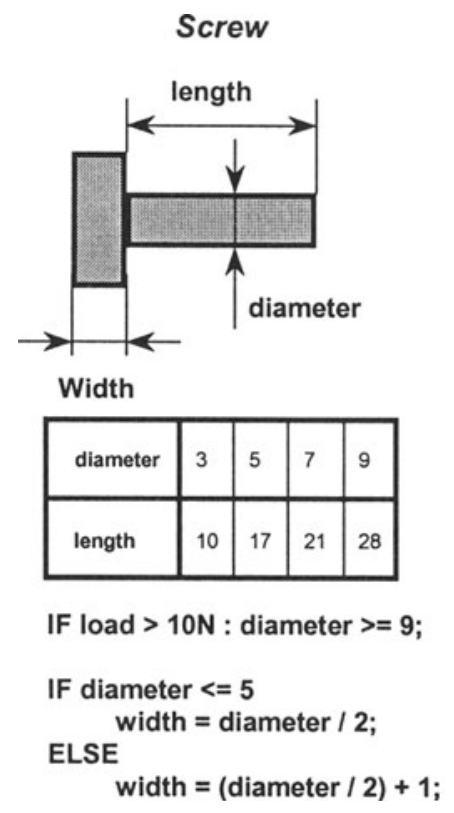

(a) temperature meter

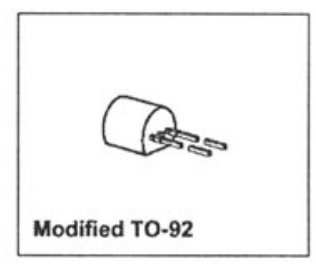

\begin{tabular}{|l|l|c|c|c|}
\hline \multicolumn{1}{|c|}{ Type } & \multicolumn{1}{|c|}{$\begin{array}{c}\text { Part } \\
\text { number }\end{array}$} & Package & $\begin{array}{c}\text { Voltage } \\
\text { max (V) }\end{array}$ & Temperature \\
\hline KT 100 & Q62705-K331 & TO-92 & 15 & 25 \\
KT 10-5 & Q62705-K110 & TO-92 & 15 & 25 \\
KT 10-6 & Q62705-K132 & TO-92 & 15 & 25 \\
KT 10-62 & Q62705-K71 & TO-92 & 15 & 25 \\
KT 10-7 & Q62705-K111 & TO-92 & 15 & 25 \\
\hline
\end{tabular}

Figure 1. Implicit and explicit representation of a family of components

Originally, PLIB provided mainly the implicit representation. Due to the requirements from MERCI, the explicit representation has been much more strengthened in the standard, and it has been fully implemented in the IS version of PLIB [ISO 13584/24].

In addition to the parametric data which are modelled directly by PLIB, there exist hooks to connect the supplemental information around a 
component in a well defined way. Thus, it is possible to use other standards in combination with the PLIB kernel and to provide this design data to the customers in the most appropriate format.

The PLIB standard has been defined formally by means of an EXPRESS information model [ISO 10303/11], which has been developed and standardised in the context of the STEP project (Standard for the exchange of product model data, (ISO10303)). This allows the automatic check of data with respect to the model and with respect to the dictionary.

\subsection{Use of dictionaries}

One central element of the PLIB standard is the use of dictionaries to clearly identify the elements used in the database. In general, a dictionary is a hierarchy of component classes. Associated to a class are the properties by which the components in this class are described, and properties are inherited along the hierarchy. Both for classes and properties, several kinds of information are provided to uniquely identify the meaning of the elements and its representation in databases (e.g. by the data type of properties). In a database or exchange file, the single components and their property values always refer to dictionary entries which describe their meaning and interpretation of codings.

For the domain of electronic components, a reference dictionary based on these concepts has been defined by the IEC [IEC61360/4]. This dictionary was supposed to be widely used, but unfortunately, the IEC claimed an intellectual property right on the dictionary without defining clearly the way how it can be used. As a result, various groups started to define and use their own dictionaries (e.g. ECALS, RosettaNet). In addition, each manufacturer uses internally his own dictionary which has been developed over a couple of years. Thus, we have to deal with a number of dictionaries.

There are two possible ways to deal with different dictionaries in a database:

1. Dictionaries can be extensions of each other. For instance, a manufacturer may choose to extend a reference dictionary (e.g. the IEC dictionary) by its own extensions.

2. Dictionaries can be independent from each other. In this case, a mapping has to be defined between the different dictionaries to make them comparable.

To handle these different situations, PLIB provides two mechanisms to combine dictionaries:

- A reference mechanism allows to connect one dictionary to another dictionary: by the "is-case-of" relationship, a class in a dictionary A can 
be linked to a class in a dictionary $B$, and it may inherit specific properties from the A-class.

- A mapping mechanism allows to map dictionaries: By the "a-posteriocase-of" relationship, classes in different dictionaries can be associated to each other and a mapping of the respective properties of these classes can be defined.

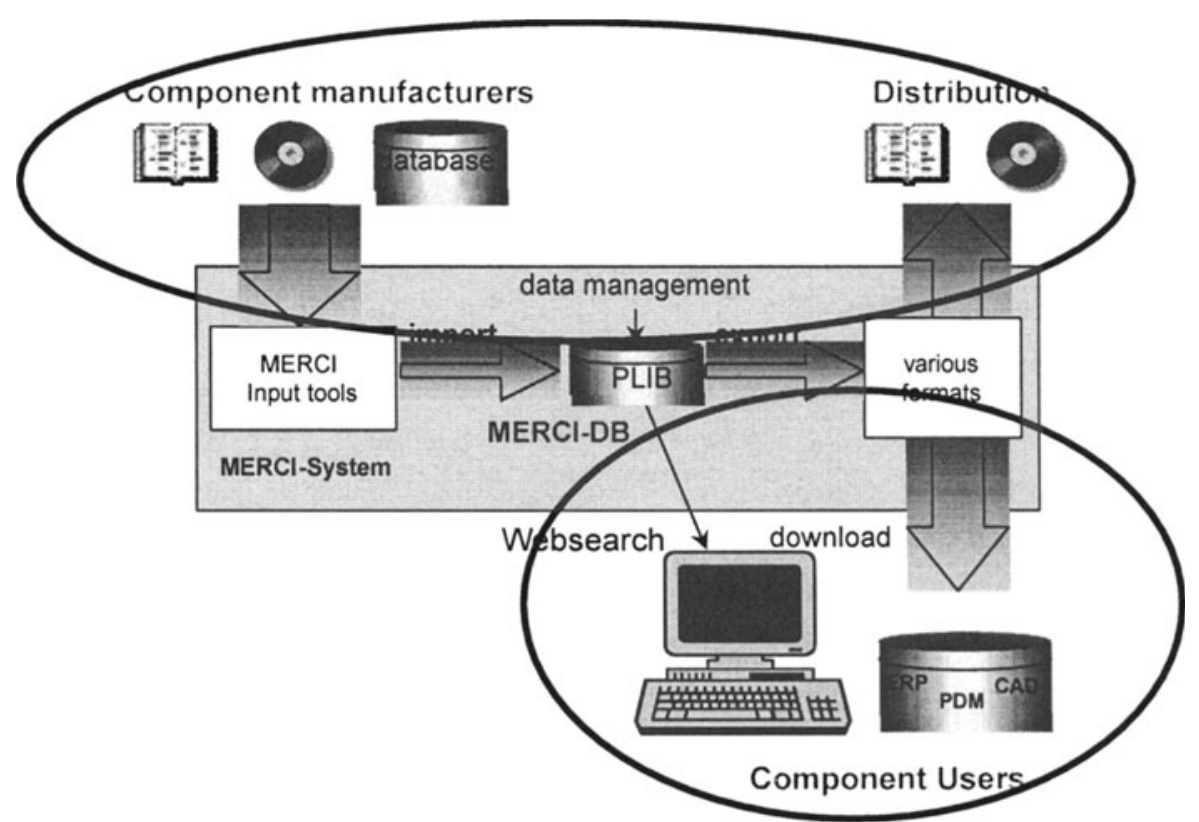

Figure 2.Overall vision of MERCI

\section{MERCI: DEALING WITH COMPONENT DATA}

\subsection{Overview and goals}

The goal of MERCI is to provide solutions and services to close the information gap between component manufactuers and component users and to support the direct flow of information between their processing systems. Therefore, MERCI provides tools to import data into the standard PLIB format and to generate data and documents in various formats from the standard PLIB format. This is illustrated in figure 2: Manufacturers provide their data in various forms, e.g. in databases, in textual data sheets, on $C D$, etc. MERCI transfers this data into the neutral PLIB format and stores the 
results in a database. This database can be used both by the component manufacturer and the component user: The manufacturer can generate various document formats from the database and thus base his publishing process on the database, and the component user can select components via the Web, download the information in various formats and document types and then integrate the downloaded data into his $\mathrm{CAD}$ libraries and corporate databases. Thus, this data is available for his further product development processes.

\subsection{Use of dictionaries in MERCI}

A central element of the MERCI system is the MERCI-DB which contains the component data and the dictionary information. In the MERCI$\mathrm{DB}$, dictionaries are used for several purposes:

- for the definition of the semantics of elements of the database

- for a hierarchical search in the component classes and for providing class specific query forms,

- for providing manufacturer independent and manufacturer specific queries

- for the database organisation of the component descriptions

For users, it is important to have the capability to search across several component manufacturers. This could be best realised by providing a common dictionary for all manufactures. On the other hand, different manufacturers like to provide different properties for their components and to use this as a means to differentiate themselves from their competitors. To fulfil both requirements, MERCI deals with dictionaries in the following way:

The central roles plays the reference dictionaries defined by the IEC. However, each manufacturer has his own dictionary with a specific entry point. But the manufacturer dictionaries are connected to the reference dictionary, i.e. they inherit most of their properties from the IEC dictionary. Only those properties are defined in the manufacturer specific part of their dictionaries which are not already part of the reference dictionary. All components are associated to a manufacturer dictionary (see figure 3 ). 


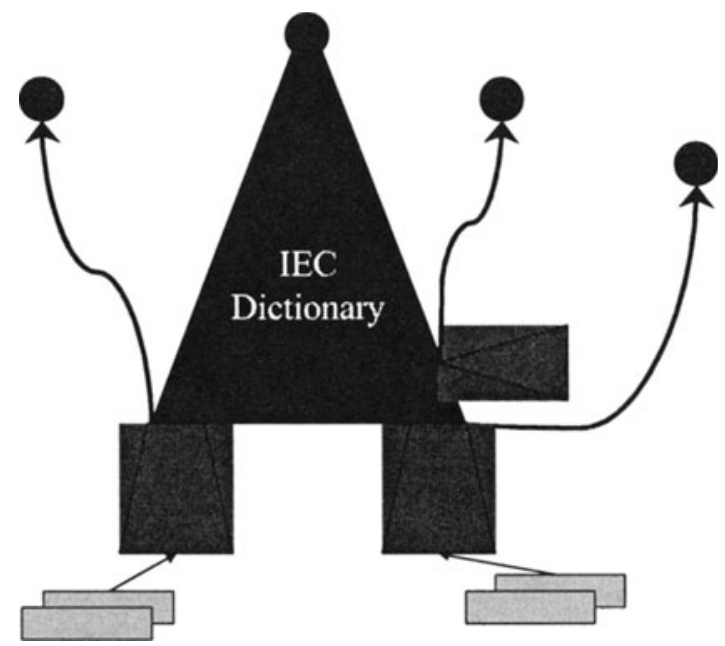

Figure 3. Dealing with dictionaries in the MERCI system

Users can use the reference dictionary for their searches across manufacturers. As long as they restrict their hierarchical search to the IEC dictionary, their query results will contain components from all available manufacturers. If they are interested to use the manufacturer specific properties for their search, then these properties are defined in the manufacturer dictionaries and are thus usable for manufacturer specific queries.

To make the dictionaries comparable, it is necessary to map their common properties to the IEC dictionary. Thus, with the import of data into the database, a mapping process needs to be initiated. The same is true for the export: Most component users also use their specific dictionaries and have to map data which they download accordingly. Thus, both import and export is accompanied by mapping processes, as illustrated in figure 4 . But again, dictionaries help: The mapping is defined on the level of the dictionary elements, and the mapping programs on the data level are then generated automatically.

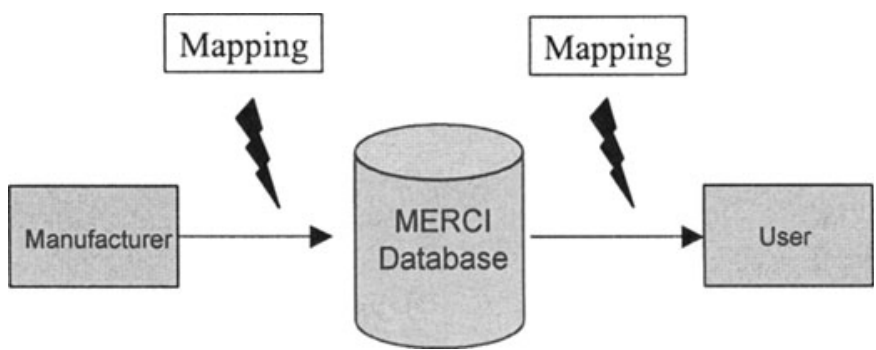

Figure 4. Import and export with mapping to dictionaries 


\subsection{Architecture of the MERCI system}

The overall architecture of the MERCI system is sketched in figure 5.

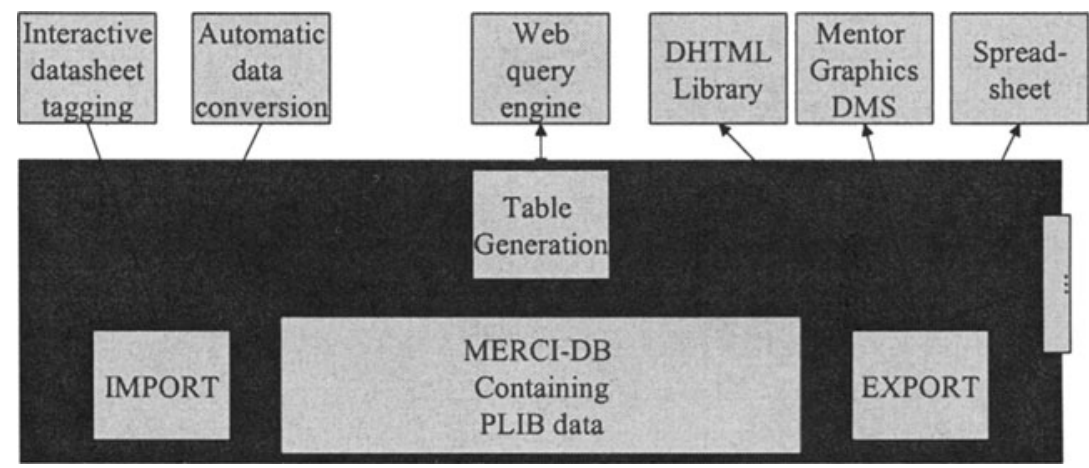

Figure 5. Overall architecture for the MERCI system

Around the PLIB compliant database, the system provides a middleware layer which contains generic mechanisms for dealing with the database. This layer is used by the tools and applications in the application layer to implement their services.

On the input side, component data can be captured. The goal is to get as much data as possible from existing sources. If these sources are structured, then it is possible to implement automatic conversion tools. But unfortunately, many of the data is kept in textual data sheets. This data can only be extracted from the data sheets in a semiautomatic process. For this purpose, a tagging tool has been implemented by MERCI which allows to associate elements of the text (like numbers) with meaning (i.e. the property which this number describes). As a result, a file is generated which contains this information in an explicit form. This serves as input for the MERCI DB.

The component data in the database can be queried via a powerful search engine through the Web. Information about selected components can be downloaded in various formats:

- Step File: This format has been defined by ISO in the context of STEP [ISO 10303/21], and it allows the exchange of data which are structured according to an EXPRESS model. This format is mainly used for the downstream import of data into a corporate database of the end user's system environment. For example, for MERCI an interface to the Mentor Graphics DMS system has been implemented. This allows the import of the downloaded STEP File into DMS from where the data can be imported into various CAD systems and a connection to PDM, ERP and BOM systems can be established. 
- EXCEL file: The tabular information about the set of components can be directly imported into EXCEL spreadsheets.

- XML can be generated according to the XML binding of STEP [ISO10303/28], according to the SimPLIB DTD [SimPLIB] and according to the RosettaNet standard.

- DHTML library format: This set of dynamic HTML files contains a full component library which comprises the data about the selected components combined with the appropriate part of the dictionary. The DHTML library provides search capabilities for selecting components, it can print order forms or it can be directly connected to a distributor channel for online ordering of components. Thus, it provides similar functionality on a local basis as the MERCI database on a global basis. It is meant as a low cost means for small and medium companies to work with the data downloaded from the MERCI DB: They get a full working library system, but the only software they need is an Internet browser.

\section{CONCLUSION AND OUTLOOOK}

The paper has shown how the MERCI system exploits the PLIB standard and associated dictionaries for developing a system which allows a more efficient exchange of component data between component manufacturers and component users. The system will support the manufacturers to switch from documents as their source of information to data as the primary source - documents can then be regarded just as a representation forms of data. Based on this philosophy, both the publishing processes can be streamlined and better organised and the information exchange with customers can be improved.

The MERCI project will finish in the first half of 2002, and it is planned to install afterwards a commercial service for manufacturers and users of components providing tools and services for data organisation, document and Web publishing and data exchange. The current prototype and demonstrator can be visited on the Web under www.merci-project.net.

\section{ACKNOWLEDGEMENTS}

Part of this work has been funded by the EU under the IST project MERCI (IST-1999-12238). The author likes to thank the partners in the MERCI project for their contributions. Partners in the project are Adepa [F], EADS (Aerospatiale Matra Missile) [F], ENSMA/LISI [F], EPM 
Technologies [N], Infineon [D], Rosemann \& Lauridsen [D], Descon / Mentor Graphics [D], and University of Hagen [D]. The data conversion tool has been implemented by Rosemann \& Lauridsen, the tagging tool and the DHTML library generator has been implemented by ENSMA/LISI, and the integration facility into the DMS system has been implemented by Descon / Mentor Graphics. The MERCI database developed at the University of Hagen is based on the EXPRESS Data Manager of EPM.

\section{REFERENCES:}

[IEC61360/4] IEC 61360-4: Standard data element types with associated classification scheme for electric components - Part 4: IEC reference collection of standard data element types, component classes and terms, 1997

[ISO13584/24] ISO/IS 13584-24: Parts library : Logical Resources : Logical model of supplier library. 2001.

[ISO13584/42] ISO/IS 13584-42: Parts library: Description methodology: Methodology for structuring parts families. 1997.

[ISO10303/11] ISO IS 10303-11: EXPRESS Language Reference Manual. 1994

[ISO10303/21] ISO IS 10303-21: Clear Text encoding of the exchange structure. 1994

[ISO10303/28] ISO CD/TS 10303/28: XML representation for EXPRESS driver data. 2001

[SimBLIB] Pierra, G.; Potier, J.-C.; Sardet, E.: From digital libraries to electronic catalogues for engineering and manufacturing. International Journal of Computer Application in Technology, July 2000. 